\title{
Student interpretations of partial derivatives
}

\author{
Paul J. Emigh and Corinne A. Manogue \\ Department of Physics, Oregon State University, Corvallis OR 97331
}

\begin{abstract}
We present results from an investigation into how students interpret partial derivatives at different points in their undergraduate career. We gave a long-answer survey to students that asked them to explain the meaning of the derivative in three different contexts. The survey was given near the beginning of a multivariable calculus class and at the start and end of a year-long junior-level physics sequence. We found two common overarching interpretations of the derivative: one corresponding to "slope" and the other to "change." We discuss the results using a concept image framework based on the work of Zandieh. We also note differences in the response patterns of the students in the mathematics and physics courses and differences in how students interpret the derivative across different representations of functions.
\end{abstract}

\section{INTRODUCTION}

Physics is filled with functions of multiple variables, so the derivatives used in physics are often partial derivatives, in which some variables are held constant. While partial derivatives are relevant to nearly all of physics, different physics subfields tend to use, calculate, and interpret them in very different ways. For example, in electromagnetism the variables tend to be spatial, such as Cartesian $(x, y, z)$ or spherical coordinates $(r, \theta, \varphi)$. In thermodynamics, the variables (e.g., pressure and entropy) are not spatial, and it is common to find partial derivatives with respect to one variable while holding virtually any other variable(s) constant. Furthermore, it is not possible in thermodynamics to hold all other variables constant. These practices do not always match how partial derivatives are first introduced in multivariable calculus. Thus, there is a need to explore student understanding of partial derivatives, and especially to identify how student thinking changes over time and how it differs in various mathematics and physics contexts.

Mathematics education researchers have previously examined student understanding of derivatives [1-2]. One particularly relevant example is Zandieh's theoretical framework [3], which uses Vinner's concept image [4] to characterize student ideas about derivatives, and which has been extended to become more relevant for research in physics contexts [5-6]. In addition, physics education researchers have begun investigating student understanding of derivatives in introductory physics courses [7-8] and in a few upper-division physics courses [9-10].

In this article, we present results from an investigation into progressions of student ideas about partial derivatives in calculus and upper-division physics. We focus on students near the beginning of two different courses: multivariable calculus and upper-level electro-magnetism. Our primary goal is to identify student interpretations of partial derivatives. To address this goal, we examined student interpretations of ordinary derivatives as well as partial derivatives. Of particular interest are differences both in the interpretations associated with different representations of multivariable functions and between students belonging to each population of interest.

We focus in this article on student understanding in mathematical contexts (that is, without a physical context). This research provides a benchmark for student thinking about partial derivatives prior to upper-division physics instruction, laying the groundwork for comparing student interpretations at different instructional stages and observing patterns or changes over time. It may also guide instructors and curriculum developers looking to improve upper-level physics courses by revealing what students think about derivatives at the start of their classes.

We begin in section II by describing our methods for examining student thinking. Section III gives the different interpretations of partial derivatives we identified. In section IV, we discuss our results from a theoretical perspective influenced by Zandieh. Lastly, we discuss implications for instructors and researchers in section IV.

\section{METHODS AND ANALYSIS}

This study was conducted with students enrolled in three courses at Oregon State University (OSU). The first was honors multivariable calculus (MC), the first math course covering partial derivatives. The MC students were mostly freshmen. The next was junior-level electromagnetism (EM), the first physics course in which students use partial derivatives. Most students in the EM course had previously completed MC, vector calculus, and ordinary differential equations. The last course was central forces (CF), the culmination of the junior-year physics sequence. Virtually all students in CF previously completed the EM course.

We gathered data by administering a written survey in class. It was given during week 1 of $\mathrm{MC}$, week 2 of EM, and week 2 of $\mathrm{CF}$, and took about 10 minutes of class time. The questions for each course were identical. In MC, the survey was given prior to instruction on partial derivatives, and thus probes student knowledge given only singlevariable calculus concepts. In EM, the survey was given 
Answer the questions below using 1-2 complete sentences. You may also support your written answer with graphs or equations.

1. For a function $h$, the derivative of $h$ with respect to $x$ is sometimes known as a rate of change. Explain the meaning of rate of change in this context.

2. During a previous class period, you were provided with a plastic surface, which is a representation of a function $h(x, y)$. Explain the meaning of the derivative of $h$ with respect to $x$ in this context.

3. The contour graph represents a function $h(x, y)$. Explain the meaning of the derivative of $h$ with respect to $x$ in this context.

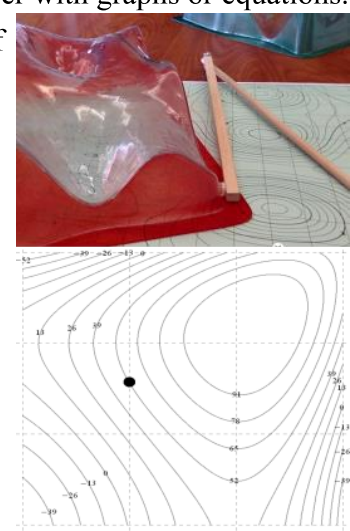

FIG 1. Three questions given as part of a written survey The plastic surface (used in all courses) is from the Raising Calculus to the Surface Project (see Ref. [11]).

before partial derivatives were used in physics contexts, to serve as a pretest for the junior-level physics sequence. The survey was given in $\mathrm{CF}$ as a corresponding post-test, after students have made substantial use of partial derivatives in EM and thermodynamics courses during the junior year. Thus, many students took the survey twice (in both EM and $\mathrm{CF}$ ). No students from MC were in either EM or CF. The survey was developed after examining several preliminary surveys in an effort to probe student thinking more reliably.

The survey consists of three questions about derivatives (see Fig. 1). It is intended to probe students' thinking about derivatives rather than their ability to compute a derivative. For example, question 1 asks students to explain what "rate of change" means in the context of a derivative. This term was chosen because we found that, in preliminary versions of the survey that simply asked them to explain the meaning of a derivative, students often used the term without further description. Questions 2 and 3 focus on what meaning students ascribe to the derivative of a multivariable function in different contexts. Question 2 uses a three-dimensional surface and question 3 uses a contour graph, both of which students handled as part of a review exercise [11].

Each question asks about a derivative with respect to $x$. We intentionally do not specify what (if anything) should be held constant. We use the word "derivative" rather than $h^{\prime}, d h / d x$, or $\partial h / \partial x$ so that our choice of notation would not impact student answers. Students are asked to use complete sentences, graphs, and/or equations.

We used open coding to characterize all statements in the student responses relevant to derivatives. The codes were then examined to identify patterns in how students interpreted the derivative. Interpretations with similar language and/or underlying structure were categorized according to the underlying meaning that those responses ascribed to the derivative. Responses consistent with more than one interpretation were classified into all relevant categories - that is, our categories are not exclusive.

\section{STUDENT INTERPRETATIONS}

In this section, we present the interpretations we identified and their overall prevalence in each course. The number of students in each course was relatively small: 19 in MC, 30 in EM, and 29 in CF (22 took the survey in both EM and CF). Therefore, we do not statistically compare the results, though some apparent differences in how the groups view derivatives may be worthy of more extensive study.

We found two predominant interpretations applicable to both ordinary and partial derivatives. The results are summarized in Table I. Our description of the results focuses first on question 1, as the percentages do not change substantially from question to question. We note that the same students did not always give identical responses across the entire survey: some answered the three questions similarly while others answered differently. After describing the two primary interpretations, we discuss four student descriptions of how to hold something constant when explaining the derivatives in questions 2 and 3 .

Interpretations: Many students identified the derivative with slope, an interpretation that has been discussed at length in prior work [2-3,7-8]. On question $1,53 \%$ of the MC students gave this interpretation. However, "slope" was far less common among physics students: only $17 \%$ in EM and $28 \%$ in CF. Although use of the term slope suggests that the interpretation is graphical, we found that only about half of the students who gave it supported their answer with a graph. The following response, from an $\mathrm{MC}$ student, was typical: "Rate of change is the slope of the function at any point along line $h . "$ The level of detail surrounding the word "slope" was highly variable, regardless of whether the response was verbal or graphical. For example, it was relatively uncommon for students to give a more detailed account of what the slope was or how it might be found. It was also rare for students to describe the slope in relation to a tangent line; instead, they tended to write about the slope of the function directly. On questions 2 and 3, a small number of students used the word "steep" or "steepness" to refer to the derivative, which also suggests to us a slope interpretation. However, only one student used "steep" together with "slope" in the same response.

The second prevalent interpretation was a tendency to make an equivalence between the derivative and the change in the given function. More physics students (70\% in EM and $82 \%$ in $\mathrm{CF}$ ) than math students $(37 \%)$ gave such a response. These students primarily used words and symbols; it was rare for them to include a graph with such

TABLE I. Student interpretations of derivatives for the MC $(\mathrm{N}=19)$, EM $(\mathrm{N}=30)$, and $\mathrm{CF}(\mathrm{N}=29)$ courses. The numbers represent percentages that are not exclusive.

\begin{tabular}{c|ccc|ccc|ccc}
\hline Interpr & \multicolumn{3}{|c|}{ Question 1 } & \multicolumn{3}{c|}{ Question 2 } & \multicolumn{3}{c}{ Question 3 } \\
-etation & MC & EM & CF & MC & EM & CF & MC & EM & CF \\
\hline Slope & 53 & 17 & 28 & 42 & 27 & 20 & 42 & 17 & 20 \\
Change & 37 & 70 & 82 & 37 & 60 & 60 & 37 & 47 & 66 \\
\hline
\end{tabular}


an interpretation. The following example (from an EM student) was a typical way of phrasing this idea: "The rate of change expresses how much $h$ changes as $x$ changes." The phrase "how much" was especially common, though some students instead referred to the derivative as "the change in $h . "$ Most of these students also referenced a change in $x$, but a few did not explicitly mention $x$ at all. In response to questions 2 and 3 , students often used language similar to their initial response to question 1 , and a few stated explicitly that the same interpretation was valid (often with some additional information about what to hold constant-see below).

What to hold constant: The categories discussed above are equally applicable to ordinary and to partial derivatives. One important extra step that must be taken when finding a partial derivative is identifying what is held constant. This step is not always a straightforward one, particularly in physics contexts [9-10,12]. We focus on questions 2 and 3, since very few responses to question 1 were relevant. We found four common approaches, summarized in Table II.

The first approach was simply stating that $y$ is held constant when taking the derivative. For example, an EM student wrote that the derivative in question 2 means "how does $h$ change as $x$ is wiggled and $y$ is constant." Since the questions indicate that $h$ is a function of $x$ and $y$, this is not surprising. The percentage of the students who gave this answer appeared to increase with more instruction, which may indicate an increase in familiarity with the common language associated with partial derivatives.

A few students gave answers that emphasized that only $x$ should be changed for the derivative. For example, a $\mathrm{CF}$ student answered question 3 by stating that the derivative is "how much the distance between contour lines changes as $x$ (and only $x$ ) is changed by a given amount." However, this was not a prevalent response in any class, and about half of the students who gave it also gave one of the other descriptions for how to hold something constant.

Many students described the derivative as being "in the $x$-direction" or "along the $x$-axis." In response to question 2 , few of these students drew a graph, but their language suggests visual or graphical thinking. Although these students did not explicitly identify some quantity to hold constant, they did so implicitly by providing a path for the derivative to follow in the $x y$-plane. It should be noted that

TABLE II. Student responses to questions 2 and 3 related to what is held constant for the MC $(\mathrm{N}=19)$, EM $(\mathrm{N}=30)$, and $\mathrm{CF}(\mathrm{N}=29)$ course. As in Table $\mathrm{I}$, the numbers represent percentages and the categories are not exclusive.

\begin{tabular}{c|ccc|ccc}
\hline \multirow{2}{*}{ Interpretation } & \multicolumn{3}{|c|}{ Question 2 } & \multicolumn{3}{c}{ Question 3 } \\
& MC & EM & CF & MC & EM & CF \\
\hline Hold $y$ constant & 16 & 33 & 41 & 5 & 3 & 24 \\
Only change $x$ & 0 & 7 & 7 & 11 & 3 & 7 \\
Direction/axis & 32 & 43 & 38 & 11 & 53 & 48 \\
Cross-section & 11 & 3 & 3 & 16 & 10 & 0 \\
\hline No discussion & 26 & 17 & 21 & 47 & 20 & 10 \\
\hline
\end{tabular}

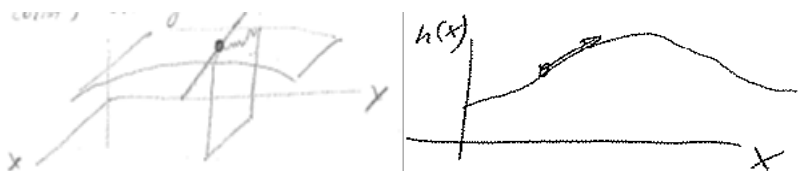

FIG 2. Sketches drawn in response to question 2 (left) and 3 (right) showing the "cross-section" mode of thinking about what to hold constant for a partial derivative.

a derivative with respect to $x$ can be taken "in a direction" other than the direction in which $y$ is constant. Such derivatives are common in thermodynamics but are substantially less common in spatial contexts.

The final idea expressed by students was more strongly visual: using a cross-section to create a new function of one dimension and then finding the resulting derivative. Some students sketched an example cross section, as shown on the left of Fig. 2. Others described the process of finding a cross-section in words, with some also drawing an example of the resulting one-dimensional function, as shown on the right of Fig. 2. This response was the least common, given by fewer than $16 \%$ of the students in each case.

Few physics students (in either EM or CF) did not describe holding something constant. More students at the beginning of multivariable calculus ( $26 \%$ on question 2 and $47 \%$ on question 3) did not discuss this idea. Such responses tended to be very similar to the responses to question 1. Since the MC students had not studied partial derivatives yet, while the EM and CF students had, this result is not necessarily surprising. Only one student found a total derivative rather than a partial derivative.

\section{THEORETICAL PERSPECTIVE - CONCEPT IMAGE}

The primary theoretical perspective that has influenced the analysis and interpretation of our results is Zandieh's concept image framework for derivatives [3]. A concept image is defined as all properties and mental pictures related to a concept. Zandieh's framework, which was developed to describe students' ideas about ordinary derivatives, organizes those properties along two dimensions: representations and process-object layers. Below, we briefly describe each dimension and some of the insight it provided to us in evaluating students' responses.

Representations are contexts within which a derivative may be understood; Zandieh's are graphical, verbal, physical, and symbolic. For contexts related to physics, we have found it useful to add a numerical representation, which refers to treating the derivative as a ratio of small (but not infinitesimal) changes $[6,13]$. This is particularly relevant because physics often deals with real-world data for which differences cannot be taken as arbitrarily small.

We found that student interpretations tended to be primarily verbal or symbolic in nature. Even students who described the derivative using slope often did not draw a picture or provide a visual connection to their answer. 
Students who wrote instead about change, including the vast majority of physics students in both $\mathrm{EM}$ and $\mathrm{CF}$, rarely made any reference to graphs. We found this to be true across all three questions on the survey, even when students were prompted with a visual representation of a function.

Process-object layers, based on Sfard [14], refer to mathematical processes acting on mathematical objects that have previously been established. For example, division can be thought of as a process that acts on two numbers (objects). The process is then reified into a new object that can be acted on by other processes (e.g., division produces a fraction that can then be treated as a single quantity). Zandieh describes the derivative in terms of three such layers: ratio, limit, and function. Each layer begins as a process that becomes an object to be acted upon by the process in the next layer. We focus here on the ratio and limit layers, as these are the most relevant to physics.

Few students discussed the ratio process-object layer, whether they interpreted the derivative as slope or change. Although many students wrote $d h / d x$, very few indicated that they thought of $d h / d x$ as division. It is thus possible that $d h / d x$ is simply familiar notation for the derivative, rather than a ratio. Similarly, almost no students discussed an explicit limit. A few used the words "instantaneous," "infinitesimal," or "small" to characterize the derivative.

At first glance, our results suggest that students' concept images of the derivative may be incomplete, even after a year of junior-level physics. Instead of directly equating the derivative with the change in a function, we would like students to think of the derivative as a "ratio of small changes" that also encompasses the ratio and limit processobject layers. Only three students (all in CF!) gave a response including all of these elements. We do not necessarily think the absence of language related to ratios or limits in students' written responses indicates they lack any knowledge of these aspects, but we are increasingly coming to believe that this may be the case for some. We have conducted interviews with students on this subject that will allow us to explore their ideas more extensively. Whether or not the language students use is indicative of a deep misunderstanding of the nature of derivatives, we believe it is valuable to devote instructional resources to enriching students' concept image by giving them a starting point that is more reflective of the complicated layers that make up the derivative. This is particularly useful when students proceed to partial derivatives, where the new layer of what to hold constant is added to the already complex concept image.

\section{DISCUSSION AND IMPLICATIONS}

One of the primary goals of this study is to describe student understanding of partial derivatives as a guide to improving instruction in upper-level physics. Our results are a first step toward characterizing how student learning about partial derivatives progresses through undergraduate math and physics courses. We identified two common interpretations of the derivative-slope and change-along with some interesting patterns in the student responses. We are in the process of conducting further research, including interviews, to deepen our understanding of student thinking.

Foremost, it appears that more physics majors view the derivative as related to change than to slope. We have examined several textbooks used for introductory calculus and calculus-based physics, including those in use at OSU, in an attempt to identify possible sources for this language. The use of slope appears to be widespread in both the calculus and the physics texts. Language similar to the change interpretation, however, was absent from the texts surveyed. We find this surprising because "how much $h$ changes" strikes us as a familiar description of derivatives in physics classrooms (introductory and upper-division) and among physics experts. We intend to survey math and physics instructors to gain insight into this interpretation.

The learning of partial derivatives is a fundamental goal of OSU's upper-division physics program. One strategy we use to foster this learning is a focus on the coordination of partial derivatives across multiple representations. The results of this study indicate that there is still room to improve how students think of and talk about ordinary derivatives as building blocks toward a more complete understanding of more advanced partial derivatives.

\section{ACKNOWLEDGEMENTS}

We thank all current and past members of the Paradigms in Physics research project, especially Tevian Dray, Elizabeth Gire, and David Roundy. We also acknowledge the students who participated in the research. This work was supported in part by NSF grant DUE-1323800.

[8] W.M. Christensen \& J.R. Thompson, Phys. Rev. ST Phys. Ed. Res., 8 (2012).

[9] D. Roundy et al., Am. J. Phys., 82 (1), 39-46 (2014).

[10] J.R. Thompson et al., PERC 2005 (2005).

[11] See https://raisingcalculus.winona.edu/.

[12]D. Roundy et al., Phys. Rev. ST Phys. Ed. Res., 11 (2015).

[13] T. Dray et al., PRIMUS (accepted).

[14] A. Sfard, Ed. Studies in Math., 22 (1991). 